\title{
Irregular X-ray Variation of LMC X-3
}

\author{
H. C. Pan ${ }^{1,3}$, G. K. Skinner ${ }^{1}$, R. A. Sunyaev ${ }^{2}$, N. L. Alexandrovich ${ }^{2}$
}

1 School of Physics and Space Research, University of Birmingham, Edgbaston, Birmingham B15 2TT, UK

2 IKI, Space Research Institute, Profsoyuznaya, 84/32, Moscow 117296, Russia

3 Department of Physics (Theoretical Physics), University of Oxford, 1 Keble Road, Oxford OX1 3NP, UK

\section{Introduction}

LMC X-3 is an X-ray binary in the Large Magellanic Cloud. It was discovered by $U H U R U$ and observations with various satellites showed that the $\mathrm{X}$-ray source was variable by a factor of up to 100 (e.g. Treves et al. 1988). Using the GINGA and $H E A O-1$ observations, Cowley et al. (1991) found a strong 198 (or possibly $\sim 99$ ) day modulation in the X-ray luminosities of LMC X-3. They suggested that this modulation may be due to an accretion disc precession, or periodic variations in the mass transfer rate, or a combination of both.

We observed LMC X-3 with the TTM in 1988-1990. The present paper gives some results from the analysis of the data obtained.

\section{Observations and Results}

The $T T M$ is a coded mask telescope on board the MIR space station. It is capable of producing images in the energy range $2-30 \mathrm{keV}$ with a spectral resolution of about $18 \%$ at $6 \mathrm{keV}$ (Brinkman et al. 1985).

We monitored LMC X-3 with the TTM in the periods 1988 November 13 -1989 March 2, 1989 May 30-June 14, and 1990 December 22. During the observations of 1988 November 13-December 11 , while the hard X-ray (7-28 keV) flux of LMC X-3 remained almost constant at about 1.5 counts $\mathrm{s}^{-1}$, the $2-7 \mathrm{keV}$ flux decreased gradually by $74 \%$ in 28 days. Although the X-ray spectrum on 1988 December 11 was not well determined owing to the source weakening, the analysis of the other spectra indicates that it is likely that LMC X-3 underwent a transition from a high to a low state in this period. LMC X-3 became bright again on 1989 January 3-6 and on around March 2 the 2-7 keV flux reached the highest level $14.7 \pm 0.3$ counts $\mathrm{s}^{-1}$ ( $\left.55 \mathrm{mCrab}\right)$, more than a factor of 8 larger than that observed on 1988 December 11. In 1989 May-June, the source flux in the 2-7 keV range was back to the level of 1988 November 13; however, the 7-28 $\mathrm{keV}$ flux was generally higher.

We have folded the $2-10 \mathrm{keV}$ fluxes of LMC X-3 from the TTM measurements with the ephemeris given by Cowley et al. (1991). We plot the folded light curve 
in Fig. 1. It is clear that the TTM observations on 1988 November 13-15, 1989 May 30-June 6, and 1990 December 22 follow the 198 day modulation. However, the observations made from 1988 November 29 to March 2 with the TTM, and also with the GINGA LAC (on 1988 December 12 and 1989 February 10, see Fig. 1), indicate that the source showed large irregular variations with the intensity rapidly decreasing from 1988 November 29 and gradually increasing from at least 1989 January 4 until March. In this period the 198 day modulation law was largely violated by LMC X-3. If the 198 day modulation reflects the precession of the accretion disc, it is possible that the $\mathrm{X}$-ray variation in the period 1988 November 29 to 1989 March 2 is caused by the change of the mass accretion rate. As the hard $\mathrm{X}$-rays showed little change in this period, we assume that the variation of the soft $\mathrm{X}$-rays (and the temperature of the soft X-ray emitting region) is directly related to the change in the mass accretion rate.

The TTM spectra of LMC X-3 can be described with a model consisting of a multi-temperature disc blackbody (Mitsuda et al. 1984) and a power-law. We find that both components are variable and there is no correlation between them.

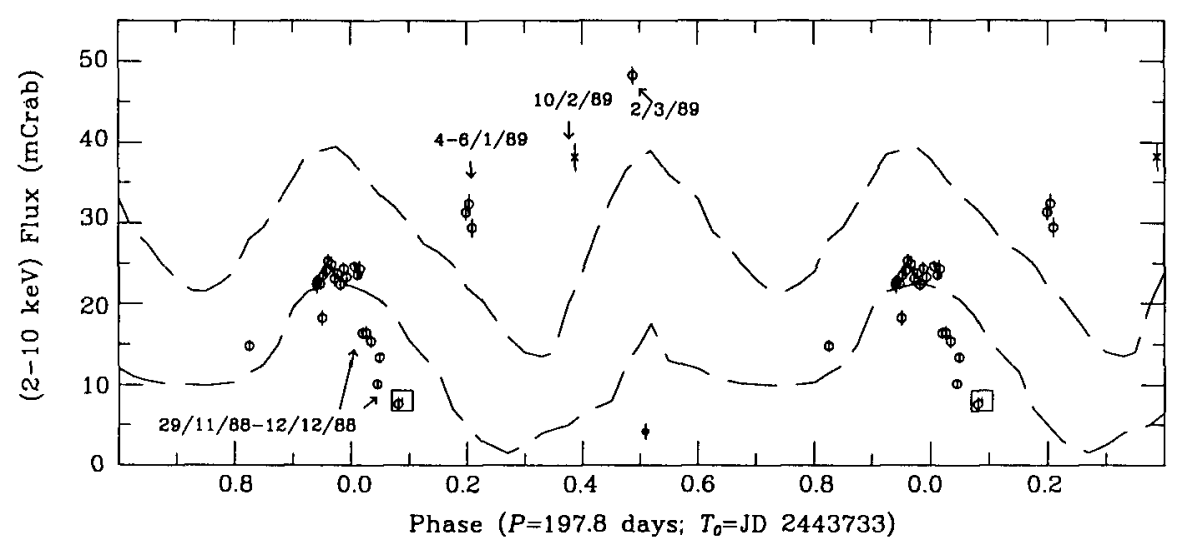

Fig. 1. X-ray (2-10 keV) intensities of LMC X-3 folded with the ephemeris of Cowley et al. (1991). The open circles represent the TTM measurements. The open squares and crosses are the GINGA fluxes. The dashed lines illustrate the 198 day modulation.

\section{References}

Brinkman A. C., Dam J., Mels W. A., Skinner G. K., Willmore P. W., 1985, in Perola G. C., Salvati M., eds, Non-thermal and Very High Temperature Phenomena in X-Ray Astronomy, Roma: Università "La Sapienza", p. 263

Cowley A.P., et al., 1991, ApJ 381, 526

Mitsuda K., et al., 1984, PASJ 36, 741

Treves A., et al., 1988, ApJ 325, 119 